Adeneye et al., Afr J Tradit Complement Altern Med. (2014) 11(2):402-410

http://dx.doi.org/10.4314/ajtcam.v11i2.26

\title{
EVALUATION OF THE ANTI-ARTHRITIC ACTIVITY OF THE HYDROETHANOLIC LEAF EXTRACT OF ALCHORNEA CORDIFOLIA IN RATS
}

\author{
Adejuwon Adewale Adeneye $^{1^{*}}$, Adekunle Ibrahim Oreagba ${ }^{2}$, Ismail Ogunbayode Ishola ${ }^{2}$, Hassanat Adeola \\ Kalejaiye $^{2}$
}

${ }^{1}$ Department of Pharmacology, Faculty of Basic Medical Sciences, Lagos State University College of Medicine, 1-5 Oba Akinjobi Way, G.R.A, Ikeja, Lagos State, Nigeria, ${ }^{2}$ Department of Pharmacology, Therapeutics and Toxicology, Faculty of Basic Medical Sciences, College of Medicine, University of Lagos, Idi-Araba, Lagos State, Nigeria

*E-mail: adeneye2001@yahoo.com

\begin{abstract}
Background: Different decoctions of Alchornea cordifolia leaves are used by Yoruba herbalists (Southwest Nigeria) for the local treatment of ulcers, rheumatic pains, febrile convulsions, and for enhancing physical performance.

Materials and methods: In this study, the anti-arthritic effect of $100-400 \mathrm{mg} / \mathrm{kg} /$ day of the hydroethanolic leaf extract of Alchornea cordifolia (HEAC) was investigated in Complete Freund's Adjuvant (CFA)-induced arthritic rats as a way of evaluating its efficacy in the local management of arthritis. In addition, the effects of HEAC on liver and renal function parameters as well as its effect on the antioxidant enzyme system were investigated. Arthritis was induced using $0.1 \mathrm{ml}$ of $10 \mathrm{mg} / \mathrm{ml}$ of Complete Freund's Adjuvant (CFA) following $1 \mathrm{~h}$ oral pretreatment and $8^{\text {th }}$ day postarthritic induction with 100, 200 and $400 \mathrm{mg} / \mathrm{kg} / \mathrm{day}$ of HEAC and $3 \mathrm{mg} / \mathrm{kg} / \mathrm{day}$ of celecoxib as the reference drug. The anti-arthritic activity of HEAC was assessed based on the ability of HEAC to alter the paw edema diameter, body weight, full blood count, renal and liver function markers, glycoprotein, lysosomal enzymes and possible antioxidant potential in the arthritic rats.

Results: Oral pretreatment with 100,200 , and $400 \mathrm{mg} / \mathrm{kg} / \mathrm{day}$ of HEAC produced significant $(p<0.001, p<0.05$ and $p<0.01)$ reductions in the paw edema diameter in a non-dose dependent fashion in ACF-induced arthritic rats with the $100 \mathrm{mg} / \mathrm{kg} /$ day of HEAC producing the most significant antiarthritic effect. Similarly, HEAC increased hepatic GSH levels, CAT and SOD activities suggesting possible antioxidant mechanism for its antiarthritic effect.

Conclusion: Overall, results of this study lend credence to the folkloric use of water decoction of Alchornea cordifolia leaves against rheumatoid arthritis. However, further pharmacological investigations would be required at isolating and determining the active anti-arthritic molecule(s) in HEAC in the nearest future.
\end{abstract}

Key words: Complete Freund's Adjuvant, Arthritis, Hydroethanolic leaf extract, Alchornea cordifolia, Rats

List of Abbreviations: AAAL - arthritis-attributable activity limitation; ALB - albumin; ALP - alkaline phosphatase; ALT - alanine aminotransferase; AST - aspartate aminotransferase ; BIL - bilirubin; CAT - catalase; CELE - celecoxib; CFA - Complete Freund's Adjuvant; $\mathrm{CHOL}$ - cholesterol; GSH - reduced glutathione; $\mathrm{H}_{2} \mathrm{SO}_{4}$ - tetraoxosulphate (VI) acid; $\mathrm{HCl}$ - hydrochloric acid; HEAC - hydroethanolic leaf extract of Alchornea cordifolia; $\mathrm{ID}_{50}$ - median inhibitory concentration; $\mathrm{MCH}$ - mean cell hemoglobin; $\mathrm{MCHC}$ - mean cell hemoglobin concentration; $\mathrm{MCV}$ - mean cell volume; MDA - malonialdehyde; $\mathrm{NaOH}$ - sodium hydroxide; PCV - packed cell volume; PLT - platelet count; p.o. - per oral; RBC red blood cells; SOD - superoxide dismutase; subc. - subcutaneous route; TG - triglyceride; TP - total protein; WBC - white blood cell count/leukocyte count

\section{Introduction}

Rheumatoid arthritis remains the most common auto-immune inflammatory disorder, affecting $1 \%$ of the adult population worldwide, and is usually associated with progressive joint destruction (Firestein, 2003). In the United States, a Centre for Disease Control (CDC), survey based on data from 2007 - 2009 revealed that $22.2 \%$ (49.9 million) of adults aged $\geq 18$ years had self-reported doctor-diagnosed arthritis, and $9.4 \%$ (21.1 million or $42.4 \%$ of those with arthritis) had arthritis-attributable-to-activity-limitation (AAAL) and with an aging population this figure is expected to rise (Woolf and Pfleger, 2003; Centers for Disease Control and Prevention, 2010). In Nigeria, the true incidence of rheumatoid arthritis remains unknown but the crude incidence of RA in a Nigerian urban city has been estimated to be $12.3 \%$ with a female-to-male ratio of 2.4:1 and mean age of 46.9 years (Adelowo et al., 2010) although an earlier study of two rural townships of southern Nigeria reported an incidence rate of $0.0 \%$ despite the fact that $5.5 \%$ of individuals tested were positive for rheumatoid factor (Silman et al., 1993). However, till date, the aetiology of rheumatoid arthritis remains controversial and debatable but there is a consensus that its etiopathogenesis could be triggered by the interplay of both genetic and environmental factors (Ollier et al., 2001). In the treatment of the disease, non-steroidal drugs, steroidal agents and immuno-suppressants are usually used but these drugs are notorious for some unwanted effects such as gastrointestinal disorders, immunodeficiency and humoral disturbances (Ong et al., 2007). Therefore, efforts are currently channelled towards discovering and developing therapeutic agents which are more effective but of lesser toxicity profile, particularly, with prolonged use. Studies showed that in the United States, 60 - 90\% patients suffering from arthritis, particularly rheumatoid arthritis, are on one form of Complementary and Alternative Medicine or the other, predominantly herbal therapies either alone and/or with conventional treatment (Rao et al., 1999). In Nigeria, the true statistics on the use of alternative and/or complementary remedy in the local management of rheumatoid arthritis remain unknown but these figures are expected to be higher bearing in mind that over $80 \%$ the population is reliant partly or wholly on one form of complementary and alternative medicine or the other for the treatment of their ailments. In addition, the World Health Organization (WHO) estimates that nearly $80 \%$ of the world population depend partly or wholly on traditional medicine, especially medicinal plants, for their primary health care needs (World Health Organization, 1996). 


\section{http://dx.doi.org/10.4314/ajtcam.v11i2.26}

Alchornea cordifolia (Schumach. and Thonn.) Müll. Arg. (family: Euphorbiaceae) is a shrub widely distributed along the coastal regions of West Africa where decoctions made from its pulverized leaves are used for the local treatment of rheumatic pains, fever, wounds and diarrhea (Ogungbamila and Samuelsson, 1999; Tona et al., 2000). The plant is commonly known as the Christmas bush. It is known by other names such as dove wood (English), Arbre de djeman (French), Bugi-bugi, bunce, pô d'arco (Portuguese). Its local names include ewe eepa, esinsin, and eepa (in Yoruba, Southwest Nigeria) (Burkill, 1997; Adewunmi et al., 2001). The rural populace of Southwest Nigeria also employs the decoction of the leaves in the management of febrile convulsions in children (Ogungbamila and Samuelsson, 1990). Previous studies have established the spasmolytic (Ogungbamila and Samuelsson, 1990), anti-inflammatory (Manga et al., 2004; Mavar-Manga et al., 2008), anti-microbial (Okeke et al., 1999; Ebi, 2001), anti-diarrhea (Tona et al., 2000, Agbor et al., 2004), and analgesic properties of the extract of Alchornea cordifolia (Osadebe and Okoye, 2003). Other studies have reported the hepato-protective and anti-oxidant effects of Alchornea cordifolia (Olaleye et al., 2006, Olaleye and Rocha, 2008), as well as its anti-anaemic effect which was mediated via increased circulating hemoglobin and iron levels (Kapnang-Jepang, 1997).The ethanol extracts of the leaf and fruit showed significant trypanocidal, anti-helminthic and amoebicidal activities (Tona et al., 2000; Adewunmi et al., 2001). Phytochemical studies of the leaf extract also revealed the presence of flavonoid, alkaloids, saponins, phenols and tannins (Ogungbamila and Samuelsson, 1999; Tona et al., 2000). In addition, the leaf extract of Alchornea cordifolia is reported to contain alchornine, alchorneinone, gentisnic acid and yohimbine (Ogungbamila and Samuelsson, 1999).

Decoctions of Alchornea cordifolia leaves remain highly valued by Yoruba herbalists (Southwest Nigeria) and it is reputedly used in the local management of arthritis. Despite its ancestral use, however, there are no pharmacological data to either confirm or refute the therapeutic potential of this plant in the management of arthritis. In view of the above, the present study was designed to evaluate the possible anti-arthritic activity and possible mechanism(s) of action of 100,200 and $400 \mathrm{mg} / \mathrm{kg} /$ day of the hydro-ethanolic leaf extract of Alchornea cordifolia against CFA-induced arthritic rats. The chosen doses of Alchornea cordifolia extract used in this study were based on results of preliminary study conducted before the main experiment (unpublished).

\section{Materials and methods \\ Collection and identification of Alchornea cordifolia leaves}

Fresh leaves of Alchornea cordifolia were collected from the deciduous forest of Abafadu Village, Ikire, Osun State, Nigeria. Botanical identification and authenticated was done by Mr. T.K. Odewo of the Herbarium, Department of Botany, University of Lagos, Akoka, Lagos State, Nigeria. A voucher specimen with number LUH 4846 was deposited in the herbarium of same department for reference purpose.

\section{Preparation of hydroethanolic leaf extract of Alchornea cordifolia}

The freshly harvested leaves of Alchornea cordifolia plant were gently but thoroughly washed under a running tap water and completely airdried for one week under shade. The dried leaves were then pulverized into fine powder using laboratory hammer mill. $400 \mathrm{~g}$ of the powder was soaked in $10 \mathrm{~L}$ of $75 \%$ hydroethanolic solution (consisting of $7.5 \mathrm{~L}$ of "absolute" ethanol and 2.51 of distilled water) for five days after which the preparation was filtered using the Whatmann's filter paper size $-9 \mathrm{~cm}$. The filtrate was evaporated in a rotary evaporator attached to a thermochiller (Buchi $700^{\circledR}$, Recirculation chiller) at a temperature of $40{ }^{\circ} \mathrm{C}$. The residue was freeze-dried to obtain a deep-brown residue of the crude extract. This procedure was conducted for three more times and the residue were pooled into an air- and water-tight container and stored in the refrigerator at $4{ }^{\circ} \mathrm{C}$ until required for experimentation.

\section{Experimental Animals}

Forty-eight healthy young Wistar Albino female rats weighing between 156 - $171 \mathrm{~g}$ and 30 male Swiss Albino mice weighing between 15 $20 \mathrm{~g}$ were obtained from the Laboratory Animal Centre, College of Medicine, University of Lagos, Idi-Araba, Lagos State, Nigeria, and used for the study after institutional ethical approval has been obtained. The animals were allowed three weeks of acclimatization and were allowed free access to standard chow and drinking water. The animals were maintained under standard environmental conditions $\left(23-25^{\circ} \mathrm{C}, 12 \mathrm{~h} / 12 \mathrm{~h} \mathrm{light} / \mathrm{dark}\right.$ cycle $)$ and had free access to standard rodent pellet diet (Livestock Feed Plc, Lagos, Nigeria) and water. The animals were acclimatized in the laboratory conditions for a week before the commencement of the study. The experimental procedures adopted in this study were in strict compliance with the United States National Institutes of Health Guidelines for Care and Use of Laboratory Animals in Biomedical Research (1985).

\section{Experimental induction of arthritis and oral treatment of rats with HEAC}

One week before the commencement of the experiment, the rats were randomly divided into 8 groups of 6 rats per group. Arthritis was induced by intradermal injection of $0.1 \mathrm{ml}$ of Complete Freund's adjuvant (CFA) $(10 \mathrm{mg} / \mathrm{kg}$ of heated-killed Mycobacterium tuberculosis in $1 \mathrm{ml}$ paraffin oil) into the right hind paw (Ahmad et al., 2006). The treatment was given for 28 and 20 days as pretreatment and post treatment, respectively. Arthritis in rats was assessed by means of physical and biochemical measurements. The joint diameter and body weight was measured on day 0 (before injecting FCA) and every 4 days for 28 days using cotton thread method as described by Bamgbose and Noamesi (1981), in both control and treated rats. The experimental rats were randomly divided into eight groups of six rats per group and treated as follows:

Group I: $10 \mathrm{ml} / \mathrm{kg}$ of $0.9 \%$ normal saline, p.o. $+0.1 \mathrm{ml} 0.9 \%$ normal saline, $s u b c$.

Group II: $10 \mathrm{ml} / \mathrm{kg}$ of $0.9 \%$ normal saline, p.o. $+0.1 \mathrm{ml} \mathrm{CFA,} \mathrm{subc.}$

Groups III - V: oral pretreatments with 100, 200, and $400 \mathrm{mg} / \mathrm{kg}$ /day of HEAC, p.o. $+0.1 \mathrm{ml} \mathrm{CFA,} \mathrm{subc.,} \mathrm{given} 1 \mathrm{~h}$ after HEAC pretreatment Groups VI - VII: 200 and $400 \mathrm{mg} / \mathrm{kg}$ HEAC, p.o. (given 8 days post-CFA treatment) $+0.1 \mathrm{ml} \mathrm{CFA,} \mathrm{subc.}$

Group VIII: $3 \mathrm{mg} / \mathrm{kg} /$ day celecoxib, p.o. $+0.1 \mathrm{ml} \mathrm{CFA}$

\section{Blood collection and bioassays}

On the $28^{\text {th }}$ day post-arthritis induction, the rats were anaesthetized by intra-peritoneal administration of combination of $25 \%$ urethane (Sigma Chemical Co., U.S.A.) and 1\% Chloralose (Sigma Chemical Co., U.S.A.) at a dose of $1.2 \mathrm{mg} / \mathrm{kg}$ body weight. Blood samples for hematological and biochemical assays were obtained through ocular puncture and collected into EDTA-treated sample bottles and plain bottles, 
http://dx.doi.org/10.4314/ajtcam.v11i2.26

respectively. Thereafter, rats were sacrificed by cervical decapitation, a ventral longitudinal abdominal incision made and the liver and spleen were identified and immediately dissected out. These dissected organs were separately rinsed and homogenized in ice-cold $0.01 \mathrm{M}, \mathrm{Tris} \mathrm{HCl}$ buffer, $\mathrm{pH} 7.4$ to give a $10 \%$ homogenate. Liver samples were taken for determination of in vivo antioxidants (reduced glutathione (GSH), superoxide dismutase (SOD), catalase (CAT)), nitrite, and malondialdehyde (MDA).

\section{Assay of lysosomal enzymes}

Acid phosphatase was assayed using the method of King (1965) by colorimetric method, using disodium phenyl phosphate as the substrate. The enzyme activity was expressed as $\mu \mathrm{mol}$ phenol liberated/min/mg/protein.

\section{Estimation of protein-bound carbohydrate}

For glycoprotein analysis, $20 \%$ of the liver or spleen samples were defatted in hexane placed in a test tube, to which $1 \mathrm{ml}$ of $2 \mathrm{~N} \mathrm{HCl} \mathrm{was}$ added, and the tubes were sealed. Hydrolysis was completed by keeping the sealed tubes at $100{ }^{\circ} \mathrm{C}$ for $16-18 \mathrm{~h}$. After hydrolysis, the contents were neutralized with $\mathrm{NaOH}$ and made up to a known volume, and aliquots were used for glycoprotein estimation. The tissue hexose level was determined by the method of Neibes (1972). The neutralized sample was mixed with orcinol- $\mathrm{H}_{2} \mathrm{SO}_{4}$ reagent, heated at $80{ }^{\circ} \mathrm{C}$, cooled and left in the dark for 25 min. Sialic acid was determined by the method of Aminoff (1961) with modifications by Neibes (1972). The neutralized sample was mixed with 0.25 $\mathrm{M}$ periodate (in $0.1 \mathrm{~N} \mathrm{H}_{2} \mathrm{SO}_{4}$ ) and the reaction was inhibited after $30 \mathrm{~min}$ by arsenite solution. Then, thiobarbituric acid was added and the contents were heated. The pink color that developed on cooling was measured at $540 \mathrm{~nm}$.

\section{Estimation of serum liver and renal functions}

Serum albumin, total protein (TP), total cholesterol (TC), triglycerides (TG), glucose, uric acid, alkaline phosphatase (ALP), aspartate transaminase (AST), alanine transaminase (ALT) and total bilirubin as liver function parameters and serum urea and creatinine (as renal function parameters) were assayed for using Roche and Cobas commercial test kits and Roche/Hitachi 904 Automated Analyzer.

\section{Antioxidant markers}

Liver and splenic reduced glutathione (GSH) (Beutler and Kelley, 1963), superoxide dismutase (SOD) (Beauchamp and Fridovich, 1971), and catalase (CAT) activities (Goth, 1991) were assayed using standard procedures.

\section{Hematological analysis}

The full hematological parameters evaluated include red blood cell (RBC) count, hemoglobin (Hb), platelet count (PLT), total and differential white blood cell (WBC) count, neutrophil, \%neutrophil, mean cell hemoglobin concentration (MCHC), mean red cell volume (MCV), and mean cell hemoglobin (MCH), and packed cell volume (PCV) were assayed for using Roche and Cobas commercial test kits and Roche/Hitachi 904 Automated Analyzer.

\section{Statistical Analysis}

Results were expressed as mean \pm SD and statistical analysis was performed using Turkey's Multiple Comparison and Bonferroni post tests using GraphPad Prism 5 (GraphPad Software Inc., California, U.S.A.). Significant values were considered at $p<0.05, p<0.01$ and $p<0.001$.

\section{Results \\ \% Yield of HEAC}

Hydroethanolic extraction of Alchornea cordifolia leaves yielded $11.06 \%(\mathrm{w} / \mathrm{w})$ of the original plant material extracted. The crude extract obtained after complete dryness was a deep-brown, sweet-smelling solid residue.

\section{Effect of HAEC on paw edema in CFA-induced arthritis in rats}

Subcutaneous injection of CFA into the right hind paw produced an increase in the right paw diameter which was maximum on day $4(0.92$ $\pm 0.15 \mathrm{~cm}$ ) but insignificantly decreased $(p>0.05)$ to $0.80 \pm 0.28 \mathrm{~cm}$ on day 28 (Table 1$)$. Oral pretreatment with $100 \mathrm{mg} / \mathrm{kg} / \mathrm{day}$ of $\mathrm{HEAC}$ initially produced an insignificant $(p>0.05)$ increase in paw diameter, $1.18 \pm 0.04 \mathrm{~cm}$ on day 4 but became significantly $(p<0.001)$ reduced to $0.34 \pm 0.08 \mathrm{~cm}$ on day 28 . With oral pretreatment with $200 \mathrm{mg} / \mathrm{kg} /$ day of HEAC, there was a significant $(p<0.05)$ decrease in the paw edema diameter on day 4 from $0.70 \pm 0.09 \mathrm{~cm}$ to $0.41 \pm 0.00 \mathrm{~cm}$ on day 28 while oral pretreatment with $400 \mathrm{mg} / \mathrm{kg} /$ day of HEAC caused a significant ( $\mathrm{p}<0.01)$ reduction in the paw edema diameter from $1.20 \pm 0.16 \mathrm{~cm}$ on day 4 to $0.36 \pm 0.02 \mathrm{~cm}$ on day 28 (Table 1). However, the maximum significant $(p<0.001)$ reduction in the paw edema diameter was produced by $100 \mathrm{mg} / \mathrm{kg} / \mathrm{day}$ of HEAC and the degree of reductions in the paw edema diameter at this oral dose was comparable to that induced by $3 \mathrm{mg} / \mathrm{kg} /$ day of celecoxib (Table 1 ).

In the post-arthritic model, oral treatment with $200 \mathrm{mg} / \mathrm{kg} /$ day of HEAC was associated with a significant $(p<0.05)$ decrease in the paw circumference from $1.15 \pm 0.03 \mathrm{~cm}$ on day 8 to $0.37 \pm 0.07 \mathrm{~cm}$ on day 28 (Table 1). Similarly, oral treatment with $400 \mathrm{mg} / \mathrm{kg} / \mathrm{day}$ of HEAC caused a significant $(p<0.05)$ decrease from $1.00 \pm 0.14 \mathrm{~cm}$ on day 8 to $0.38 \pm 0.03 \mathrm{~cm}$ on day 28 and this significant reduction in paw edema was similar to that caused by $3 \mathrm{mg} / \mathrm{kg} / \mathrm{day}$ of celecoxib (from $1.10 \pm 0.04 \mathrm{~cm}$ on day 8 to $0.40 \pm 0.04 \mathrm{~cm}$ on day 28) (Table 1). 
http://dx.doi.org/10.4314/ajtcam.v11i2.26

\section{Effect of $100-400 \mathrm{mg} / \mathrm{kg} / \mathrm{day}$ HEAC on body weight changes in CFA-arthritic rats}

Repeated daily subcutaneous injection of $0.1 \mathrm{ml}$ of CFA was associated with non-significant $(p>0.05)$ time dependent increase in the weight changes in CFA-induced arthritic rats over the 28-days treatment period (Table 2). Oral pre-treatment with $100 \mathrm{mg} / \mathrm{kg} / \mathrm{day}$ of HEAC caused significant $(p<0.01, p<0.01, p<0.001)$ time dependent increase in the body weight changes on days 20,24 and 28 , respectively, while similar significant $(p<0.05, p<0.01, p<0.01)$ body weight changes were also recorded for $400 \mathrm{mg} / \mathrm{kg} / \mathrm{day}$ of HEAC (Table 2). However, oral pre-treatment with $200 \mathrm{mg} / \mathrm{kg} / \mathrm{day}$ of HEAC caused no significant alterations in body weight changes in the treated rats (Table 2).

In the post-arthritic rats, oral treatment with $400 \mathrm{mg} / \mathrm{kg} /$ day of HEAC caused significant $(p<0.05, p<0.01)$ time related increase in the body weight changes on days 24 and 28 of treatment, an effect that was similar to that caused by oral treatment with $3 \mathrm{mg} / \mathrm{kg} / \mathrm{day}$ of celecoxib (Table 2 ). Again, there were no significant alterations in the body weight changes at $200 \mathrm{mg} / \mathrm{kg} / \mathrm{day}$ of HEAC over the same treatment period (Table 2).

Table 1: Effect of 100-400 mg/kg oral treatment of Alchornea cordifolia hydroethanolic leaf extract on paw edema in CFA-induced arthritic rats

\begin{tabular}{|l|l|l|l|l|l|l|l|}
\hline Treatment & Day 4 (cm) & Day 8 $(\mathrm{cm})$ & Day 12 $(\mathrm{cm})$ & Day16 $(\mathrm{cm})$ & Day 20 $(\mathrm{cm})$ & Day 24 (cm) & Day 28 (cm) \\
\hline $10 \mathrm{ml} / \mathrm{kg} \mathrm{DW}$ & $0.92 \pm 0.15$ & $0.53 \pm 0.03$ & $0.80 \pm 0.10$ & $0.50 \pm 0.06$ & $0.50 \pm 0.06$ & $0.50 \pm 0.09$ & $0.80 \pm 0.28$ \\
\hline $100 \mathrm{mg} / \mathrm{kg}$ HEAC (Pre) & $1.18 \pm 0.04$ & $0.66 \pm 0.02$ & $0.56 \pm 0.14$ & $0.40 \pm 0.09$ & $0.38 \pm 0.10$ & $0.36 \pm 0.09$ & $0.34 \pm 0.08^{\mathrm{c}}$ \\
\hline $200 \mathrm{mg} / \mathrm{kg}$ HEAC (Pre) & $0.70 \pm 0.09$ & $0.48 \pm 0.09$ & $0.58 \pm 0.15$ & $0.45 \pm 0.03$ & $0.45 \pm 0.03$ & $0.45 \pm 0.03$ & $2.1 \pm 0.00^{\mathrm{a}}$ \\
\hline $400 \mathrm{mg} / \mathrm{kg}$ HEAC (Pre) & $1.20 \pm 0.16$ & $0.52 \pm 0.05$ & $0.52 \pm 0.05$ & $0.42 \pm 0.02$ & $0.40 \pm 0.00$ & $0.40 \pm 0.00$ & $0.36 \pm 0.02^{\mathrm{b}}$ \\
\hline $200 \mathrm{mg} / \mathrm{kg}$ HEAC (Post) & $1.15 \pm 0.03$ & $0.60 \pm 0.04$ & $0.60 \pm 0.04$ & $0.58 \pm 0.05$ & $0.48 \pm 0.09$ & $0.40 \pm 0.06$ & $0.37 \pm 0.07^{\mathrm{b}}$ \\
\hline $400 \mathrm{mg} / \mathrm{kg}$ HEAC (Post) & $1.00 \pm 0.14$ & $0.48 \pm 0.05$ & $0.45 \pm 0.03^{\mathrm{a}}$ & $0.40 \pm 0.00$ & $0.40 \pm 0.00$ & $0.38 \pm 0.03$ & $0.38 \pm 0.03^{\mathrm{b}}$ \\
\hline $3 \mathrm{mg} / \mathrm{kg} \mathrm{CELE}$ & $1.10 \pm 0.04$ & $0.55 \pm 0.06$ & $0.45 \pm 0.03^{\mathrm{a}}$ & $0.43 \pm 0.05$ & $0.43 \pm 0.05$ & $0.43 \pm 0.05$ & $0.40 \pm 0.04^{\mathrm{b}}$ \\
\hline
\end{tabular}

Table 2: Effect of 100-400 mg/kg of Alchornea cordifolia hydroethanolic leaf extract on body weight changes (g) of CFA-induced arthritic rats

\begin{tabular}{|l|l|l|l|l|l|l|l|}
\hline Treatment & $4^{\text {th }}$ day $\Delta \mathrm{Wt}$ & $8^{\text {th }}$ day $\Delta \mathrm{Wt}$ & $12^{\text {th }}$ day $\Delta \mathrm{Wt}$ & $16^{\text {th }}$ day $\Delta \mathrm{Wt}$ & $20^{\text {th }}$ day $\Delta \mathrm{Wt}$ & $24^{\text {th }}$ day $\Delta \mathrm{Wt}$ & $28^{\text {th }}$ day $\Delta \mathrm{Wt}$ \\
\hline Control & $7.67 \pm 3.37$ & $12.70 \pm 4.42$ & $17.00 \pm 4.50$ & $21.30 \pm 4.66$ & $26.30 \pm 8.95$ & $38.30 \pm 8.71$ & $36.80 \pm 8.34$ \\
\hline $100 \mathrm{mg} / \mathrm{kg}$ HEAC $($ Pre $)$ & $8.05 \pm 1.80$ & $8.26 \pm 1.80$ & $11.80 \pm 8.85$ & $51.00 \pm 15.80^{\mathrm{a}}$ & $75.40 \pm 17.30^{\mathrm{c}}$ & $84.60 \pm 15.20^{\mathrm{c}}$ & $96.00 \pm 15.50^{\mathrm{c}}$ \\
\hline $200 \mathrm{mg} / \mathrm{kg}$ HEAC $($ Pre) & $6.75 \pm 0.85$ & $7.50 \pm 1.70$ & $20.80 \pm 6.17$ & $33.80 \pm 9.78$ & $35.50 \pm 10.70$ & $41.30 \pm 12.20$ & $51.30 \pm 13.30^{\mathrm{a}}$ \\
\hline $400 \mathrm{mg} / \mathrm{kg}$ HEAC $($ Pre $)$ & $14.00 \pm 4.34$ & $23.40 \pm 3.97$ & $30.80 \pm 4.57$ & $44.60 \pm 7.46^{\mathrm{a}}$ & $64.40 \pm 6.74^{\mathrm{b}}$ & $74.60 \pm 5.18^{\mathrm{c}}$ & $85.40 \pm 5.56^{\mathrm{c}}$ \\
\hline $200 \mathrm{mg} / \mathrm{kg}$ HEAC $($ Post) & $8.80 \pm 1.16$ & $11.20 \pm 3.93$ & $13.60 \pm 7.78$ & $18.60 \pm 11.80$ & $32.20 \pm 12.50$ & $40.20 \pm 13.30$ & $47.00 \pm 14.00$ \\
\hline $400 \mathrm{mg} / \mathrm{kg}$ HEAC $($ Post) & $8.60 \pm 1.44$ & $21.20 \pm 5.88$ & $34.60 \pm 10.20^{\mathrm{a}}$ & $42.20 \pm 12.00^{\mathrm{a}}$ & $53.80 \pm 15.40^{\mathrm{b}}$ & $61.40 \pm 15.20^{\mathrm{a}}$ & $70.80 \pm 15.40^{\mathrm{b}}$ \\
\hline $3 \mathrm{mg} / \mathrm{kg}$ CELE & $8.67 \pm 1.12$ & $23.30 \pm 2.85$ & $33.70 \pm 4.98^{\mathrm{a}}$ & $56.20 \pm 7.35^{\mathrm{b}}$ & $51.80 \pm 12.60^{\mathrm{b}}$ & $60.80 \pm 12.90^{\mathrm{a}}$ & $68.20 \pm 12.90^{\mathrm{b}}$ \\
\hline
\end{tabular}

Table 3: Effect of 100-400 mg/kg of hydroethanolic leaf extract of Alchornea cordifolia on the full blood count of CFA-induced arthritic rats

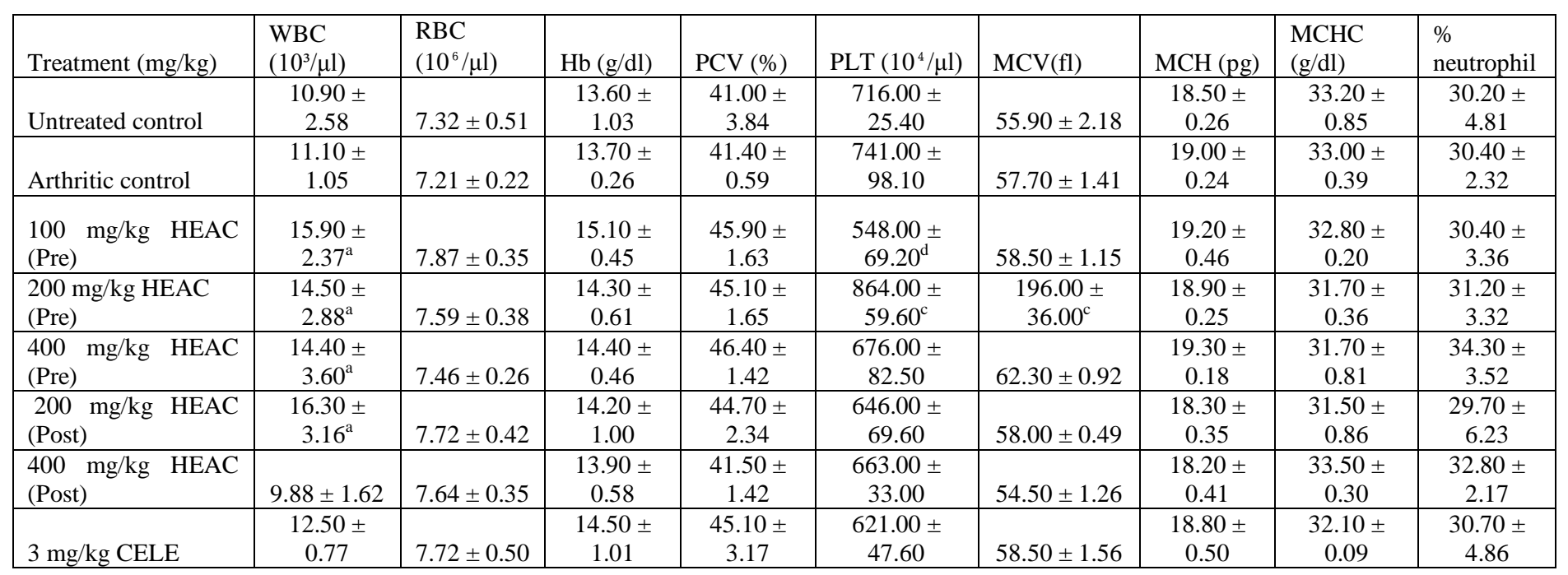


Adeneye et al., Afr J Tradit Complement Altern Med. (2014) 11(2):402-410

http://dx.doi.org/10.4314/ajtcam.v11i2.26

Table 4: Effect of oral pretreatment with $100-400 \mathrm{mg} / \mathrm{kg}$ HEAC on liver function parameters of CFA-induced arthritic rats

\begin{tabular}{|c|c|c|c|c|c|c|c|c|c|c|c|}
\hline Treatment & AST (U/l) & $\begin{array}{l}\text { BIL } \\
(\mathrm{mg} / \mathrm{dl}) \\
\end{array}$ & $\begin{array}{l}\text { CREA } \\
(\mathrm{mg} / \mathrm{dl})\end{array}$ & & ALT (U/l) & $\begin{array}{l}\text { UREA } \\
(\mathrm{mg} / \mathrm{dl})\end{array}$ & $\begin{array}{l}\text { ALB } \\
(\mathrm{mg} / \mathrm{dl})\end{array}$ & $\begin{array}{l}\text { TP } \\
(\mathrm{mg} / \mathrm{dl})\end{array}$ & $\begin{array}{l}\text { CHOL } \\
(\mathrm{mg} / \mathrm{dl})\end{array}$ & TG & ALP \\
\hline Untreated control & $\begin{array}{l}320.00 \quad \pm \\
51.40\end{array}$ & $\begin{array}{l}5.33 \quad \pm \\
0.14\end{array}$ & $\begin{array}{l}72.20 \\
8.76 \\
\end{array}$ & & $\begin{array}{l}59.50 \\
12.00\end{array}$ & $9.35 \pm 1.27$ & $\begin{array}{l}40.00 \quad \pm \\
2.81\end{array}$ & $\begin{array}{l}81.40 \quad \pm \\
1.33\end{array}$ & $2.51 \pm 0.17$ & $\begin{array}{l}0.97 \pm 0 . \\
11\end{array}$ & $\begin{array}{l}173.00 \pm 34 \\
40\end{array}$ \\
\hline Control arthritic & $\begin{array}{l}390.00 \\
39.50^{\mathrm{a}}\end{array}$ & $\begin{array}{l}4.45 \\
0.21 \\
\end{array}$ & $\begin{array}{l}55.50 \\
2.27^{\mathrm{d}} \\
\end{array}$ & \pm & $\begin{array}{l}64.80 \quad \pm \\
1.91\end{array}$ & $9.40 \pm 0.45$ & $\begin{array}{ll}41.10 \quad \pm \\
1.06\end{array}$ & $\begin{array}{l}76.60 \quad \pm \\
1.94\end{array}$ & $2.11 \pm 0.13$ & $\begin{array}{l}0.80 \pm 0 . \\
20\end{array}$ & $\begin{array}{l}215.00 \pm 32 . \\
70^{\mathrm{a}}\end{array}$ \\
\hline $\begin{array}{l}100 \mathrm{mg} / \mathrm{kg} \text { HEAC } \\
(\text { Pre })\end{array}$ & $\begin{array}{l}304.00 \\
19.50 \\
\end{array}$ & $\begin{array}{l}4.35 \\
0.21 \\
\end{array}$ & $\begin{array}{l}54.60 \\
1.65^{\mathrm{d}} \\
\end{array}$ & \pm & $\begin{array}{l}67.30 \\
7.40 \\
\end{array}$ & $8.58 \pm 0.59$ & $\begin{array}{l}42.70 \\
0.81 \\
\end{array}$ & $\begin{array}{l}77.20 \quad \pm \\
2.09 \\
\end{array}$ & $2.47 \pm 0.14$ & $\begin{array}{l}0.95 \pm 0 . \\
11\end{array}$ & $\begin{array}{l}209.00 \pm 59 . \\
10\end{array}$ \\
\hline $\begin{array}{l}200 \mathrm{mg} / \mathrm{kg} \text { HEAC } \\
\text { (Pre) }\end{array}$ & $\begin{array}{l}397.00 \\
28.40^{\mathrm{a}} \\
\end{array}$ & $\begin{array}{l}4.85 \\
0.07 \\
\end{array}$ & $\begin{array}{l}55.70 \\
2.16^{\mathrm{d}} \\
\end{array}$ & \pm & $\begin{array}{l}59.90 \\
1.66 \\
\end{array}$ & $\begin{array}{l}10.00 \quad \pm \\
0.53\end{array}$ & $\begin{array}{ll}41.70 \quad \pm \\
1.55 & \\
\end{array}$ & $\begin{array}{l}76.60 \quad \pm \\
1.45\end{array}$ & $2.37 \pm 0.24$ & $\begin{array}{l}0.61 \pm 0 . \\
07\end{array}$ & $\begin{array}{l}256.00 \pm 42 . \\
00^{\mathrm{b}}\end{array}$ \\
\hline $\begin{array}{l}400 \mathrm{mg} / \mathrm{kg} \text { HEAC } \\
\text { (Pre) }\end{array}$ & $\begin{array}{l}319.00 \\
21.30 \\
\end{array}$ & $\begin{array}{l}4.83 \\
0.21 \\
\end{array}$ & $\begin{array}{l}55.50 \\
2.71^{\mathrm{d}} \\
\end{array}$ & \pm & $\begin{array}{l}57.10 \\
4.74 \\
\end{array}$ & $8.20 \pm 0.78$ & $\begin{array}{ll}43.90 \quad \pm \\
2.41 & \\
\end{array}$ & $\begin{array}{l}76.80 \quad \pm \\
2.72 \\
\end{array}$ & $2.37 \pm 0.07$ & $\begin{array}{l}0.74 \pm 0 . \\
13\end{array}$ & $\begin{array}{l}332.00 \pm 46 . \\
20^{\mathrm{c}}\end{array}$ \\
\hline $\begin{array}{l}200 \mathrm{mg} / \mathrm{kg} \text { HEAC } \\
\text { (Post) }\end{array}$ & $\begin{array}{l}339.00 \\
8.28 \\
\end{array}$ & $\begin{array}{l}4.43 \\
0.19 \\
\end{array}$ & $\begin{array}{l}51.40 \\
3.37^{\mathrm{d}} \\
\end{array}$ & \pm & $\begin{array}{l}64.00 \\
3.80 \\
\end{array}$ & $8.90 \pm 0.65$ & $\begin{array}{l}40.30 \quad \pm \\
1.66\end{array}$ & $\begin{array}{l}70.40 \quad \pm \\
1.44\end{array}$ & $2.54 \pm 0.36$ & $\begin{array}{l}0.63 \pm 0 . \\
03\end{array}$ & $\begin{array}{l}259.00 \pm 44 \\
50^{\mathrm{b}}\end{array}$ \\
\hline $\begin{array}{l}400 \mathrm{mg} / \mathrm{kg} \text { HEAC } \\
\text { (Post) }\end{array}$ & $\begin{array}{l}306.00 \\
25.50\end{array}$ & $\begin{array}{l}4.25 \\
0.13\end{array}$ & $\begin{array}{l}50.90 \\
1.13^{\mathrm{d}} \\
\end{array}$ & \pm & $\begin{array}{l}55.60 \\
5.87 \\
\end{array}$ & $8.30 \pm 0.65$ & $\begin{array}{l}42.00 \\
2.40 \\
\end{array}$ & $\begin{array}{l}75.00 \quad \pm \\
3.13\end{array}$ & $2.08 \pm 0.17$ & $\begin{array}{l}0.95 \pm 0 . \\
09\end{array}$ & $\begin{array}{l}258.00 \pm 48 \\
50^{\mathrm{b}}\end{array}$ \\
\hline $3 \mathrm{mg} / \mathrm{kg}$ CELE & $\begin{array}{l}325.00 \\
26.40\end{array}$ & $\begin{array}{l}4.73 \\
0.43 \\
\end{array}$ & $\begin{array}{l}51.50 \\
5.03^{\mathrm{d}}\end{array}$ & 1 & $\begin{array}{l}57.90 \quad \pm \\
4.24\end{array}$ & $7.58 \pm 0.55$ & $\begin{array}{l}39.90 \quad \pm \\
2.82\end{array}$ & $\begin{array}{l}73.70 \quad \pm \\
3.67\end{array}$ & $2.43 \pm 0.10$ & $\begin{array}{l}0.77 \pm 0 . \\
17\end{array}$ & $\begin{array}{l}292.00 \pm 20 . \\
70^{\mathrm{c}}\end{array}$ \\
\hline
\end{tabular}

$\mathrm{a}, \mathrm{b}$ and ${ }^{\mathrm{c}}$ represent significant increases at $\mathrm{p}<0.05, \mathrm{p}<0.01$ and $\mathrm{p}<0.001$, respectively, when compared to untreated control group values and ${ }^{\mathrm{d}}$ represents a significant decrease at $\mathrm{p}<0.05$ when compared to untreated control group

\begin{tabular}{|c|c|c|c|c|c|c|c|c|c|}
\hline Treatment & $\begin{array}{l}\mathrm{GSH} \\
\text { protein) }\end{array}$ & $\begin{array}{l}\mathrm{SOD}(\mathrm{U} / \mathrm{mg} \\
\text { protein) }\end{array}$ & $\begin{array}{l}\text { CAT }(\mathrm{U} / \mathrm{mg} \\
\text { protein) }\end{array}$ & $\begin{array}{l}\text { MDA (ng/mg } \\
\text { protein) }\end{array}$ & Nitrite & $\begin{array}{l}\text { Hexose } \\
\text { sugar }\end{array}$ & Sialic & $\begin{array}{l}\text { Acid } \\
\text { phosphatase } \\
(\mathrm{U} / \mathrm{l})\end{array}$ & $\begin{array}{l}\text { Prostatic } \\
\text { phosphatase } \\
(\mathrm{U} / \mathrm{l})\end{array}$ \\
\hline Untreated control & $1.38 \pm 0.20$ & $1.89 \pm 0.16$ & $13.27 \pm 2.67$ & $0.02 \pm 0.00$ & $\begin{array}{ll}8.62 & \pm \\
1.24 & \end{array}$ & $\begin{array}{ll}522.90 \quad \pm \\
10.17\end{array}$ & $\begin{array}{ll}199.70 \quad \pm \\
2.58\end{array}$ & $\begin{array}{ll}67.20 & \pm \\
11.77 & \end{array}$ & $60.50 \pm 13.43$ \\
\hline Arthritic control & $1.07 \pm 0.22^{\mathrm{d}}$ & $1.09 \pm 0.10^{\mathrm{d}}$ & $12.34 \pm 0.92$ & $0.09 \pm 0.00^{\mathrm{c}}$ & $\begin{array}{ll}11.17 \quad \pm \\
0.77\end{array}$ & $\begin{array}{ll}676.40 \quad \pm \\
7.49 & \\
\end{array}$ & $\begin{array}{ll}378.70 & \pm \\
5.92^{\mathrm{c}} & \\
\end{array}$ & $\begin{array}{ll}134.10 & \pm \\
10.96^{\mathrm{c}} & \\
\end{array}$ & $94.71 \pm 2.36^{\mathrm{a}}$ \\
\hline $\begin{array}{l}100 \quad \mathrm{mg} / \mathrm{kg} \\
\text { HEAC (Pre) }\end{array}$ & $1.19 \pm 0.15^{\mathrm{a}}$ & $2.10 \pm 0.52^{\mathrm{a}}$ & $13.00 \pm 1.15$ & $0.02 \pm 0.00^{\mathrm{d}}$ & $\begin{array}{ll}7.09 & \pm \\
0.50 & \end{array}$ & $\begin{array}{ll}558.90 \quad \pm \\
16.93 & \end{array}$ & $\begin{array}{ll}338.80 & \pm \\
4.30^{\mathrm{b}} & \end{array}$ & $\begin{array}{ll}76.02 & \pm \\
18.68^{\mathrm{e}} & \end{array}$ & $53.93 \pm 10.61^{\mathrm{d}}$ \\
\hline $\begin{array}{l}200 \quad \mathrm{mg} / \mathrm{kg} \\
\text { HEAC (Pre) }\end{array}$ & $1.59 \pm 0.15^{\mathrm{a}}$ & $2.14 \pm 0.50^{\mathrm{a}}$ & $14.39 \pm 2.03$ & $0.02 \pm 0.00^{\mathrm{d}}$ & $\begin{array}{l}10.53 \pm \\
1.68\end{array}$ & $\begin{array}{ll}576.50 \quad \pm \\
53.33\end{array}$ & $\begin{array}{ll}199.70 & \pm \\
22.52^{\mathrm{e}} & \end{array}$ & $\begin{array}{ll}79.29 & \pm \\
2.12^{\mathrm{e}} & \\
\end{array}$ & $41.34 \pm 1.04^{\mathrm{e}}$ \\
\hline $\begin{array}{l}400 \quad \mathrm{mg} / \mathrm{kg} \\
\text { HEAC (Pre) }\end{array}$ & $1.36 \pm 0.19^{\mathrm{a}}$ & $4.00 \pm 0.20^{\mathrm{b}}$ & $15.39 \pm 2.03$ & $0.03 \pm 0.01^{\mathrm{d}}$ & $\begin{array}{ll}7.98 & \pm \\
0.67 & \end{array}$ & $\begin{array}{ll}462.00 & \pm \\
14.91^{\mathrm{e}} & \end{array}$ & $\begin{array}{ll}81.78 & \pm \\
10.11^{\text {f }} & \end{array}$ & $\begin{array}{l}63.05 \pm 10.2 \\
2^{\mathrm{e}}\end{array}$ & $68.34 \pm 3.38^{d}$ \\
\hline $\begin{array}{l}200 \quad \mathrm{mg} / \mathrm{kg} \\
\text { HEAC (Post) }\end{array}$ & $1.48 \pm 0.19^{\mathrm{a}}$ & $1.61 \pm 0.15$ & $17.03 \pm 1.00$ & $0.06 \pm 0.02$ & $\begin{array}{l}10.32 \pm \\
1.19\end{array}$ & $\begin{array}{ll}855.00 & \pm \\
71.66^{\mathrm{b}} & \end{array}$ & $\begin{array}{l}318.80 \quad \pm \\
39.54^{\mathrm{b}, \mathrm{d}}\end{array}$ & $74.23 \pm 1.80^{\mathrm{e}}$ & $63.36 \pm 0.24^{\mathrm{d}}$ \\
\hline $\begin{array}{l}400 \quad \mathrm{mg} / \mathrm{kg} \\
\text { HEAC (Post) }\end{array}$ & $2.48 \pm 0.46^{\mathrm{c}}$ & $3.03 \pm 0.60^{\mathrm{b}}$ & $15.36 \pm 2.85$ & $0.04 \pm 0.00^{\mathrm{d}}$ & $\begin{array}{l}10.76 \pm \\
0.07\end{array}$ & $\begin{array}{ll}515.50 & \pm \\
61.77^{\mathrm{d}} & \end{array}$ & $\begin{array}{ll}250.50 & \pm \\
18.68^{\text {a, d }} & \end{array}$ & $\begin{array}{l}75.28 \pm 14.6 \\
1^{\mathrm{e}}\end{array}$ & $79.05 \pm 1.08^{d}$ \\
\hline $3 \mathrm{mg} / \mathrm{kg}$ CELE & $1.66 \pm 0.27^{\mathrm{a}}$ & $1.54 \pm 0.35$ & $8.72 \pm 0.63$ & $0.02 \pm 0.002^{\mathrm{d}}$ & $\begin{array}{l}10.78 \pm \\
0.29\end{array}$ & $\begin{array}{ll}536.80 \quad \pm \\
7.86\end{array}$ & $\begin{array}{l}235.60 \\
13.97^{\mathrm{a}, \mathrm{d}}\end{array} \quad \pm$ & $42.96 \pm 7.19^{\mathrm{f}}$ & $21.75 \pm 1.06^{\mathrm{f}}$ \\
\hline
\end{tabular}

${ }^{\mathrm{a}, \mathrm{b}}$ and ${ }^{\mathrm{c}}$ represent significant increases at $p<0.05, p<0.01$ and $p<0.001$, respectively, when compared to untreated control. ${ }^{\mathrm{d}, \mathrm{e}}$ and ${ }^{\mathrm{f}}$ represent significant decreases at $\mathrm{p}<0.05, \mathrm{p}<0.01$ and $\mathrm{p}<0.001$ when compared to arthritic control values

Table 6: Effect of $100-400 \mathrm{mg} / \mathrm{kg}$ of HEAC on splenic antioxidant and glycoprotein in CFA-induced arthritic rats

\begin{tabular}{|c|c|c|c|c|c|c|c|}
\hline Treatment & GSH (U/mg protein) & $\begin{array}{l}\text { SOD } \\
\text { (U/mg protein) }\end{array}$ & $\begin{array}{l}\text { CAT } \\
\text { (U/mg protein) }\end{array}$ & $\begin{array}{l}\text { MDA } \\
\text { (ng/mg protein) }\end{array}$ & Nitrite & Hexose sugar & Sialic \\
\hline Untreated control & $0.56 \pm 0.06$ & $4.74 \pm 1.45$ & $16.51 \pm 3.54$ & $0.02 \pm 0.006$ & $2.72 \pm 0.20$ & $473.50 \pm 28.47$ & $119.60 \pm 10.46$ \\
\hline Arthritic control & $0.29 \pm 0.08^{\mathrm{d}}$ & $1.80 \pm 0.41^{\mathrm{d}}$ & $18.44 \pm 4.81$ & $0.08 \pm 0.001^{\mathrm{a}}$ & $4.48 \pm 1.70^{\mathrm{a}}$ & $528.70 \pm 98.70$ & $248.60 \pm 17.85^{b}$ \\
\hline $100 \mathrm{mg} / \mathrm{kg}$ HEAC (Pre) & $0.23 \pm 0.03^{\mathrm{d}}$ & $1.43 \pm 0.28^{\mathrm{d}}$ & $15.12 \pm 1.52$ & $0.06 \pm 0.004$ & $2.66 \pm 0.44^{\mathrm{d}}$ & $567.40 \pm 6.25$ & $90.11 \pm 9.50^{\mathrm{e}}$ \\
\hline $200 \mathrm{mg} / \mathrm{kg} \mathrm{HEAC} \mathrm{(Pre)}$ & $0.27 \pm 0.06^{\mathrm{d}}$ & $2.04 \pm 0.28^{\mathrm{a}}$ & $19.38 \pm 0.81$ & $0.03 \pm 0.005^{\mathrm{d}}$ & $2.85 \pm 0.45^{\mathrm{d}}$ & $378.50 \pm 20.21^{\mathrm{d}}$ & $79.26 \pm 2.82^{\mathrm{e}}$ \\
\hline $400 \mathrm{mg} / \mathrm{kg} \mathrm{HEAC} \mathrm{(Pre)}$ & $7.34 \pm 1.53^{b}$ & $1.55 \pm 0.09^{d}$ & $16.82 \pm 0.32$ & $0.08 \pm 0.02^{\mathrm{a}}$ & $2.66 \pm 0.28^{d}$ & $439.50 \pm 16.13^{d}$ & $93.55 \pm 0.78^{d}$ \\
\hline $200 \mathrm{mg} / \mathrm{kg}$ HEAC (Post) & $2.91 \pm 0.97^{\mathrm{a}}$ & $2.25 \pm 0.27^{\mathrm{a}}$ & $21.19 \pm 4.32$ & $0.10 \pm 0.03$ & $2.52 \pm 0.18^{\mathrm{d}}$ & $509.20 \pm 20.09$ & $67.11 \pm 9.56^{\mathrm{f}}$ \\
\hline $400 \mathrm{mg} / \mathrm{kg}$ HEAC (Post) & $11.51 \pm 0.97^{\mathrm{c}}$ & $1.76 \pm 0.35^{\mathrm{d}}$ & $18.09 \pm 3.90$ & $0.06 \pm 0.01$ & $1.54 \pm 0.00^{\mathrm{e}}$ & $259.00 \pm 23.20^{\mathrm{e}}$ & $70.97 \pm 15.38^{f}$ \\
\hline $3 \mathrm{mg} / \mathrm{kg} \mathrm{CELE}$ & $6.78 \pm 1.05^{\mathrm{b}}$ & $1.18 \pm 0.16^{\mathrm{d}}$ & $13.46 \pm 1.31$ & $0.06 \pm 0.01$ & $3.05 \pm 0.04^{\mathrm{a}}$ & $538.10 \pm 21.74$ & $147.00 \pm 10.72^{2}$ \\
\hline
\end{tabular}

${ }^{\mathrm{a}, \mathrm{b}}$ and ${ }^{\mathrm{c}}$ represent a significant increase at $p<0.05, p<0.01$ and $p<0.001$ when compared to untreated control group while ${ }^{\mathrm{d}, \mathrm{e}}$ and ${ }^{\mathrm{f}}$ represent significant decreases at $p<0.05, p<0.01$ and $p<0.001$ when compared to untreated arthritic rats. 
In the CFA-arthritic rats pretreated with $100 \mathrm{mg} / \mathrm{kg} /$ day of HEAC, there was a significant $(p<0.05)$ increase in the WBC and concomitant significant $(p<0.001)$ decrease in PLT (Table 3). Similarly, $200 \mathrm{mg} / \mathrm{kg} / \mathrm{day}$ of HEAC caused significant $(p<0.001)$ increases in PLT and MCV while 400 $\mathrm{mg} / \mathrm{kg} /$ day of HEAC caused no significant alterations in full blood count (Table 3). In the post-arthritic model, oral treatment with $200 \mathrm{mg} / \mathrm{kg} / \mathrm{day}$ of HEAC caused a significant $(\mathrm{p}<0.05)$ increase in WBC and caused no significant alterations in other measured parameters of full blood count (Table 3 ).

\section{Effect of $100-400 \mathrm{mg} / \mathrm{kg} /$ day of HEAC on liver function parameters in CFA-treated rats}

Repeated daily subcutaneous injection of $0.1 \mathrm{ml}$ of CFA caused significant $(p<0.05)$ increases in the serum AST and ALP when compared to that of untreated control group (Table 4). Oral pretreatment with $200 \mathrm{mg} / \mathrm{kg} / \mathrm{day}$ of HEAC caused significant $(p<0.05$ and $p<0.001)$ increases in the serum AST and ALP levels, respectively, while oral pretreatment at $400 \mathrm{mg} / \mathrm{kg} /$ day of HEAC caused a significant $(p<0.001)$ increase in the serum ALP (Table 4). In the post-arthritic model, oral treatment with 200 and $400 \mathrm{mg} / \mathrm{kg} /$ day of HEAC, and $3 \mathrm{mg} / \mathrm{kg} / \mathrm{day}$ of celecoxib caused significant $(p<0.001)$ in the serum ALP levels only while having no significant alterations in the serum levels of other measured liver function parameters such as TP, ALB, BIL, ALT, CHOL and TG (Table 4). On the renal function parameters, oral treatment with $100-400 \mathrm{mg} / \mathrm{kg} / \mathrm{day} \mathrm{of} \mathrm{HEAC} \mathrm{caused} \mathrm{non-}$ significant $(p>0.05)$ reductions in the serum urea and creatinine in both pre- and post-arthritic models (Table 4$).$

Effect of 100 - $400 \mathrm{mg} / \mathrm{kg} /$ day of HEAC on hepatic tissue oxidative stress markers, hepatic lysosomal enzymes and glycoproteins in CFAarthritic rats

Subcutaneous injection of $0.1 \mathrm{ml}$ of CFA caused non-significant ( $p>0.05)$, reductions in the hepatic tissue SOD, CAT and GSH levels. Although repeated subcutaneous injection of CFA caused significant increases in hepatic sialic $(p<0.001)$, and prostatic phosphate $(p<0.05)$ levels but caused non-significant $(p>0.05)$, increases in the hepatic tissue levels of MDA, nitrite, hexose sugar and acid phosphatase levels (Table 5). However, $100 \mathrm{mg} / \mathrm{kg} /$ day of HEAC caused a significant $(p<0.01)$ increase in the sialic level but equally caused a significant $(\mathrm{p}<0.001)$ decrease in prostatic phosphatase levels when compared to untreated control values (Table 5). $200 \mathrm{mg} / \mathrm{kg} / \mathrm{day}$ of HEAC also caused significant $(p<0.001)$ decreases in the sialic and prostatic phosphatase levels (Table 5). In the same vein, $400 \mathrm{mg} / \mathrm{kg} / \mathrm{day}$ of HEAC caused significant $(p<0.001)$ increases in the hepatic SOD activity while causing significant $(p<0.05, p<0.001)$ decreases in the hexose sugar and sialic levels when compared to values obtained for untreated arthritic group (Table 5).

In the post-arthritic model, oral treatment with $400 \mathrm{mg} / \mathrm{kg} / \mathrm{day}$ of HEAC caused significant $(p<0.05)$ increases in the hepatic GSH level and SOD activity and significant $(p<0.01, p<0.001)$ decreases in the hepatic hexose sugar, sialic, and prostatic phosphatase levels when compared to untreated arthritic group values (Table 5).

\section{Effect of 100 - $400 \mathrm{mg} / \mathrm{kg} /$ day of HEAC on splenic tissue oxidative stress markers and glycoproteins in CFA-arthritic rats}

In CFA-treated rats, there were significant $(p<0.05)$ decreases in the splenic GSH level and SOD activity and concomitant significant $(p<0.05, p<0.001)$ increases in MDA, nitrite and sialic levels when compared to the arthritic group values (Table 6). Oral treatment at $400 \mathrm{mg} / \mathrm{kg} / \mathrm{day}$ of HEAC caused significant $(p<0.05, p<0.001)$ increase in the splenic GSH level and MDA activity but significant $(p<0.05, p<0.001)$ decreases in the splenic SOD activity and sialic levels when compared to untreated arthritic group (Table 6). In the post-arthritic rats, 200 and $400 \mathrm{mg} / \mathrm{kg} / \mathrm{day}$ of HEAC caused significant $(p<0.01, p<0.001)$ dose-dependent increases in the splenic GSH levels and significant $(p<0.001)$ non-dose dependent decreases in the sialic levels when compared to the untreated arthritic group (Table 6).

\section{Discussion}

Complete Freund's Adjuvant-induced arthritis remains one of the oldest experimental animal models used to mimick human rheumatoid arthritis and it is typically and extensively used in the study of suspected agents with anti-arthritic potentials (Roubenoff et al., 1997). Other experimental animal models of rheumatoid arthritis include rat collagen-induced arthritis model (Kaklamarius, 1992; Stasiuk et al., 1996; Vierboom et al., 2005), mouse collagen-induced arthritis (Boissier et al., 1987; Campbell et al., 2000; Brand et al., 2007) and mouse collagen antibody-induced arthritis models (Brand, 2005). Inoculation of animals with CFA (which was prepared by suspending heat-killed Mycobacterium butyricum in liquid paraffin at a dose of $10 \mathrm{mg} / \mathrm{ml}$ Mycobacterium butyricum in paraffin oil), is known to produce a profound systemic inflammation resulting in severe joint swelling and remodeling (Omoto et al., 2005) and this is usually characterized by mechanical allodynia, thermal hyperalgesia and pain on joint movement (joint hyperalgesia) (Tatsuo et al., 1994; Jasmin et al., 1998; Bertorelli et al., 1999; Nagakura et al., 2003).

In the present study, experimental arthritis was reliably established with repeated and daily subcutaneous plantar injection of $0.1 \mathrm{ml}$ of CFA over a period of 28 days which was characterized by plantar edema formation which maximized on day 4 of the treatment and subsequently subsided for the remaining part of the study. The peak inflammation on day 4 induced by CFA is primarily due to edema formation and cellular influx (Kleinau et al., 1994). Thus, our results were similar to those reported by Rajkapoor et al. (2007) and Sumanth et al. (2012). Oral pre-arthritic treatment with $100-400 \mathrm{mg} / \mathrm{kg} / \mathrm{day}$ of HEAC and post-arthritic oral treatment with 200 and $400 \mathrm{mg} / \mathrm{kg}$ of HEAC in arthritic rats profoundly decreased paw edema formation in the CFA-induced arthritic rats, with $100 \mathrm{mg} / \mathrm{kg}$ of HEAC producing the most profound effect. The observation that $100 \mathrm{mg} / \mathrm{kg} \mathrm{HEAC}$ produced anti-edematous effect that is similar to that of the standard anti-inflammatory drug, celecoxib strongly suggests the possible antiinflammatory effect of the extract. Previous studies have reported that the aqueous and methanol leaf extracts of Alchornea cordifolia produced marked anti-inflammatory effect following oral and topical administration, respectively (Osadebe and Okoye, 2003; Mavar-Manga et al., 2004). Osadebe and Okoye (2003) have shown that the terpenoids and tannin containing fraction of the extract produced marked anti-inflammatory effect that is devoid of ulcerogenic adverse effect. It was evidenced in this study that both pre-treatment and post-treatment of rats with A. cordifolia significantly inhibited CFA-induced arthritis as well as inhibition of inflammatory markers. This was substantiated by the efficacy of celecoxib (selective cyclooxygenase II inhibitor (COX-II)) in the present study. The anti-arthritic effect of A. cordifolia was found to be comparable to that of celecoxib.

Another significant finding of the present study is the effect of CFA treatment on body weight changes of treated rats. While CFA-treated rats were observed to have reduced weight gain pattern over the treatment period which is in concordance with that previously reported by Roubenoff 
http://dx.doi.org/10.4314/ajtcam.v11i2.26

et al. (1997), pretreatment with $100-400 \mathrm{mg} / \mathrm{kg}$ of HEAC caused significant weight gain changes in the treated rats reversing the cachexic effect of CFA treatment with $100 \mathrm{mg} / \mathrm{kg}$ of the extract still causing the most significant weight gain. It is well documented in the literature that weight loss and cachexia are common features in rheumatoid arthritis and these are thought to be due to increased cytokine production which increases resting metabolic rate and protein breakdown as well as poor appetite (Yoshizaki et al., 1998; Rall and Roubenoff, 2004). Due to failure in adequately meeting the nutritional needs especially the increased calorie and protein needs, weight loss usually ensue (Gartlehner et al., 2006; Rall and Roubenoff, 2004). The fact that oral treatment with HEAC improved the weight gain pattern in the treated arthritic rats as observed in this study suggest that this could be due to improvement in the arthritic state of the rats. However, this remains an assertion until validated.

On the hematological profile, CFA-induced arthritis in rats is known to be associated with leukocytosis with relative lymphocytosis (Sumanth et al., 2012). In the present study, induction with CFA is associated with elevation in the total leukocyte count which again is in strong compliance with that previously reported by Sumanth et al. (2012) although in this study, differential lymphocyte was not done. Similarly, elevations in the total leukocyte count, PCV, Hb and MCV by oral pretreatment with $100-400 \mathrm{mg} / \mathrm{kg}$ of HEAC are suggestive of the possible anti-anemic effect of HEAC, although a study by Kapnang-Jepang (1997) has previously reported the anti-anemic effect of this plant.

On the liver enzyme markers, repeated subcutaneous CFA injection caused significant elevations in the serum AST and ALP while other measured parameters of hepatic injury were not significantly altered. AST and ALP are considered non-specific markers of acute hepatocellular injury and their elevations could be found in hepatobiliary disease (Kantharia and Woolf, 1987; Aida, 1993). This hypothesis may not be plausible in this study since other markers of hepatocellular injury such as alanine aminotransferase, total protein, total bilirubin, total cholesterol and triglyceride were not significantly altered in the arthritic rats. However, the profound elevations in the serum AST and ALP in the CFA-treated rats could also have arisen from extra-hepatic sources such as acute myocardial infarction and/or bone diseases and remodeling (Kantharia and Woolf, 1987; Aida, 1993). Thus, the profound elevation in the serum ALP in the arthritic rats strongly points to the establishment of an active bone lesion such as arthritis in the CFA-treated rats which is in complete agreement with results of other studies (Price and Sammons, 1974; Rosalki et al., 1982; Cimmino and Accardo, 1990; Cimmino et al., 1990). Further elevations in the serum levels of AST and ALP caused by oral treatment with 100-400 mg/kg of HEAC in both pre- and post-arthritic rat models could possibly have arisen from worsening of the chemically induced arthritis or remodeling phase of bone healing. However, the former is unlikely since oral treatment with HEAC has elicited anti-edematous effect in the CFA-induced arthritic rats. The non-hepatic toxicity of the extract was further corroborated by the non-significant elevations in the hepatic GSH levels and CAT activities but significant elevations in the SOD activities particularly at the oral dose of $400 \mathrm{mg} / \mathrm{kg}$ of the extract. Literature has shown that reductions in the GSH, SOD and CAT are strong and reliable markers of oxidative stress and increases in their levels are indicative of the antioxidative property (Siacic et al., 1997; Djordjevic et al., 2004; Anuradha and Krishnamoorthy, 2011). Thus, the increase in the levels of the oxidative stress markers by oral treatment with the extract is indicative of the inherent oxidative property of the extract. Also, antioxidant effects of some medicinal plants have been attributed to their rich abundance in flavonoids, phenols and alkaloids and previous reports on the phytochemical analysis of the leaf extract have shown Alchornea cordifolia leaves to contain flavonoid, alkaloids, saponins, phenols and tannins (Ogungbamila and Samuelsson, 1999; Tona et al., 2000). Therefore, the antioxidant effect of HEAC as seen in this study could be to the presence of the phytochemicals either singly or in combination with one another.

\section{Conclusion}

Overall, the result of this study shows the anti-arthritic effect of 100-400 mg/kg of HEAC which was possibly mediated via anti-edematous and anti-oxidative mechanisms. Further studies in the area of isolation and characterization of the active principle(s) responsible for the anti-arthritic effect of HEAC would be required in the nearest future.

\section{References}

1. Adelowo, O.O., Ojo, O., Oduenyi, I. and Okwara, C.C. (2010). Rheumatoid arthritis among Nigerians: the first 200 patients from a rheumatology clinic. Clinical Rheumatology 29(6): 593-597.

2. Adewunmi, C.O., Agbedahunsi, J.M., Adebajo, A.C., Aladesanmi, A.J., Murphy, N. and Wando, J. (2001). Ethno-veterinary medicine: screening of Nigerian medicinal plants for trypanocidal properties. Journal of Ethnopharmacology 77: 19-24.

3. Agbor, G.A., Leopold, T. and Jeanne, N.Y. (2004). The antidiarrheal activity of Alchornea cordifolia leaf extract. Phytotherapy Research 18: 873876.

4. Ahmad, S.F., Khan, B., Suri, K.A., Satti, N.K, and Qazi, G.N. (2006). Amelioration of adjuvant-induced arthritis by ursolic acid through altered Th1/Th2 cytokine production. Pharmacology Resources 53: 233-240.

5. Aida, S. (1993). Alkaline phosphatase isoenzyme activities in rheumatoid arthritis: hepatobiliary enzyme dissociation and relation to disease activity. Annals of the Rheumatic Diseases 52: 511-516.

6. Aminoff, D. (1961). Methods for the quantitative estimation of $\mathrm{N}$-acetyl neuraminic acid and their application to hydrolysates of sialomucoids. Biochemistry Journal 81: 384-392.

7. Anuradha, R. and Krishnamoorthy, P. (2011). Antioxidant activity of methanolic extract of Pongamia pinnata on lead acetate induced hepatic damage in rats. African Journal of Biochemistry Research 5(12): 348-351.

8. Bamgbose, S.O.A. and Noamesi, B.K. (1981). Studies on cryptolepine inhibition of carrageenan-induced edema. Planta Medica 41(4): $392-396$.

9. Beauchamp, C. and Fridovich, I. (1971). Superoxide dismutase: improved assays and an assay applicable to acrylamide gels. Analytical Biochemistry 44: 276-287.

10. Beutler, E. and Kelly, B. (1963). The effect of sodium on RBC glutathione. J. Experientia 19: 96-103.

11. Bertorelli, R., Corradini, L., Rafiq, K., Tupper, J., Calo, G. and Ongini, E. (1999). Nociceptin and ORL-1 ligand $\left[\mathrm{Phe}^{1} \psi\left(\mathrm{CH}_{2}-\mathrm{NH}\right) \mathrm{Gly}^{2}\right]$ nociception (1-13)NH2 exert anti-opioid effects in the Freund's adjuvant induced arthritic rat model of chronic pain British Journal of Pharmacology 128: 1252-1258.

12. Boissier, M.C., Feng, X.Z., Carlioz, A., Roudier, R. and Fournier, C. (1987). Experimental autoimmune arthritis in mice. I. Homologous type II collagen is responsible for self-perpetuating chronic polyarthritis. Annals of Rheumatoid Disease 46: 691-700. 
http://dx.doi.org/10.4314/ajtcam.v11i2.26

13. Brand, D.D. (2005). Rodent models of rheumatoid arthritis. Comparative Medicine 55(2): 114-122.

14. Brand, D.D., Latham, K.A. and Rosloniec, E.F., 2007. Collagen-induced arthritis. Nature Protocols 2: 1269-1275.

15. Burkill, H.M. (1994). The useful plants of West Tropical Africa. 2nd Edition. Volume 2, Families E-I. Royal Botanic Gardens, Kew, Richmond, United Kingdom. Pp. 636.

16. Campbell, I.K., Hamilton, J.A. and Wicks, I.P. (2000). Collagen-induced arthritis in C57BL/6 (H-2b) mice: new insights into an important disease model of rheumatoid arthritis. European Journal of Immunology 30: 1568-1575.

17. Centers for Disease Control and Prevention (CDC) (2010). Prevalence of doctor-diagnosed arthritis and arthritis-attributable activity limitation --United States, 2007-2009. MMWR Morbidity \& Mortality Weekly Report 59(39): 1261-1265.

18. Cimmino, M.A. and Accardo, S. (1990). Changes in the isoenzyme pattern of alkaline phosphatase in patients with rheumatoid arthritis. Clinical Chemistry 36(7): 1376-1377.

19. Cimmino, M.A., Buffrini, L., Barisone, G., Bruzzone, M. and Accardo, S. (1990). Alkaline phosphatase activity in the serum of patients with rheumatoid arthritis. Zeitschrift Rheumatologie 49(3): 143-146.

20. Djordjevic, A., Spasic, S., Jovanovic-Galovic, A., Djordjevic, R. and Grubor-Lajsic, G., (2004). Oxidative stress in diabetic pregnancy: SOD, CAT and GSH-Px activity and lipid peroxidation products. The Journal of Fetal-Maternal \& Neonatal Medicine 16(6): $367-372$.

21. Ebi, G.C. (2001). Antimicrobial activities of Alchornea cordifolia. Fitoterapia 72: 69-72.

22. Firestein, G.S. (2003). Evolving concepts of rheumatoid arthritis. Nature 423: 356-361.

23. Gartlehner, G., Hansen, R.A., Jonas, B.L., et al. (2006). The comparative efficacy and safety of biologies for the treatment of rheumatoid arthritis: a systematic review and metaanalysis. Journal of Rheumatology 33: 2398-2408.

24. Goth, L. (1991). A simple method for determination of serum catalase activity, and revision of reference range. Clin. Chim. Acta 196: 143-151.

25. Jasmin, L., Kohan, L., Franssen, M., Janni, G. and Goff, J.R. (1998). The cold plate as a test of nociceptive behaviors: description and application to the study of chronic neuropathic and inflammatory pain models. Pain 75: 367-382.

26. Kaklamanis, P.M. (1992). Experimental animal models resembling rheumatoid arthritis. Clinical Rheumatology 11(1): 41-47.

27. Kantharia, B.K., Woolf, A.D. (1987). Raised alkaline phosphatase in rheumatoid arthritis: primary biliary cirrhosis? British Journal of Rheumatology 26(4): 313-314.

28. Kapnang-Jepang, J.R. (1997). Etude de l'effet anti-anémique d'Alchornea cordifolia. Mémoire de Maîtrise en Biochimie, Université de Yaoundé, Département de Biochimie, Yaoundé, Cameroon. Pp. 26.

29. King, J. (1965). The hydrolase-acid and alkaline phosphatases. In: Practical Clinical Enzymology, Van, D., editor. Nostrand Company Limited, London. Pp. 191-208.

30. Kleinau, S., Erlandsson, H., Klareskog, L. (1994). Percutaneous exposure of adjuvant oil causes arthritis in DA rats. Clinical \& Experimental Immunology 96: 281-284.

31. Marva-Manga, H.M., Brkic, D., Marie, D.E.P., Quetin-Leclercq, J. (2004). In vivo anti-inflammatory activity of Alchornea cordifolia (Schumach. \& Thonn.) Müll. Arg. (Euphorbiaceae). Journal of Ethnopharmacology 92(2-3): 209-214.

32. Mavar Manga, H., Haddad, M., Pieters, L., Baccelli, C., Penge, A., Quetin-Leclercq, J. (2008). Anti-inflammatory compounds from leaves and root bark of Alchornea cordifolia (Schumach. \& Thonn.) Müll. Arg. Journal of Ethnopharmacology 115: 25-29

33. Nagakura, Y., Okada, M., Kohara, A., Kiso, T., Toya, T., Iwai, A., Wanibuchi, F. and Yamaguchi, T. (2003). Allodynia and hyperalgesia in adjuvant-induced arthritic rats: Time course of progression and efficacy of analgesics. Journal of Pharmacology and Experimental Therapeutics 306(2): 490-497.

34. Niebes, P. (1972). Determination of enzymes and degradation products of glycosaminoglycan metabolism in the serum of healthy and various subjects. Clin Chim Acta 42: 399-408.

35. Ogungbamila, F.O. and Samuelsson, G. (1990). Smooth muscle relaxing flavonoids from Alchornea cordifolia (leaves). Acta Pharmaceutica Nordica 2: 421-422.

36. Okeke, I.N., Ogundaini, A.O., Ogunbamila, F.O. and Lamikanra, A. (1999). Antimicrobial spectrum of Alchornea cordifolia leaf extract. Phytotherapy Research 13: 67-69.

37. Olaleye, M.T., Adegboye, O.O. and Akindahunsi, A.A. (2006). Alchornea cordifolia extract protects Wistar albino rats against acetaminopheninduced liver damage. African Journal of Biotechnology 5: 2439-2445.

38. Olaleye, M.T. and Rocha, J.B. (2008). Acetaminophen-induced liver damage in mice: Effects of some medicinal plants on the oxidative defense system. Experimental Toxicology and Pathology 59: 319-327.

39. Ollier, W.E., Harrison B. and Symmons, D. (2001). What is the natural history of rheumatoid arthritis? Baillieres Best Practices Research in Clinical Rheumatology 15: 27-48.

40. Omoto, A., Kawahito, Y., Prudovsky, I., Tubouchi, Y., Kumura, M., Ishino, H., Wada, M., Yoshida, M., Kohno, M., Yoshimura, R., Yoshikawa, T. and Sano, H. (2005). Copper chelation with tetrathiomolybdate suppresses adjuvant-induced arthritis and inflammation-associated cachexia in rats. Arthritis Research \& Therapy 7: R1174-R1182.

41. Ong, C.K.S., Lirk, P., Tan, C.H. and Seymour, R.A. (2007). An evidence-based update on non-steroidal anti-inflammatory drugs. Clinical Medicine \& Research 5(1): 19-34.

42. Osadebe, P.O. and Okoye, F.B.C. (2003). Anti-inflammatory effects of crude methanolic extract and fractions of Alchornea cordifolia leaves. Journal of Ethnopharmacology 89: 19-24.

43. Price, C.P. and Sammons, H.G. (1974). The nature of the serum alkaline phosphatases in liver diseases. Journal of Clinical Pathology 27(5): 392398.

44. Rall, L.C. and Roubenoff, R. (2004).Rheumatoid cachexia: metabolic abnormalities, mechanisms and interventions. Rheumatology 43: 12191223.

45. Rajkapoor, B., Ravichandra, V., Gobinath, M., Anbu, J., Harikrishnan, N., Sumithra, M., Sankari, M., Venugopal, R. and Sakthisekaran, D. (2007). Effect of Bauhinia variegate on Complete Freund's Adjuvant induced arthritis in rats. J Pharmacol Toxicol 2: 465-472

46. Rao, J.K., Mihaliak, K., Kroenke, K., Bradley, J., Tierney, W.M. and Weinberger, M. (1999). Use of complementary therapies for arthritis among patients of rheumatologists. Annals of Internal Medicine 131: 409-416.

47. Rosalki, S.B., Foo, A.Y. and Tanner, P.A. (1982). Serum gamma-glutamyltransferase and alkaline phosphatase in rheumatoid arthritis. Journal of Clinical Pathology 35(12): 1395-1395. 
Adeneye et al., Afr J Tradit Complement Altern Med. (2014) 11(2):402-410

http://dx.doi.org/10.4314/ajtcam.v11i2.26

48. Roubenoff, R., Freeman, L.M., Smith, D.E., Abad, L.W., Dinarello, C.A., Kehayias, J.J. (1997). Adjuvant arthritis as a model of inflammatory cachexia.and Rheumatism 40: 534-539.

49. Saicic, Z.S., Simovic, M.O., Korac, B., Blagojevic, D., Buzadzic, B., Spasic, M.B. and Petrovic, V.M. (1997). Activity of antioxidant defense enzymes and glutathione content in some tissues of the Belgrade (b/b) laboratory rat - Applications to mammalian blood and other tissues. Comparative Biochemistry and Physiology - Part C: Pharmacology, Toxicology and Endocrinology 117(2): 173-179.

50. Silman, A.J., Ollier, W., Holligan, S., Birrell, F., Adebajo, A., Asuzu, M.C., Thomson, W. and Pepper, L. (1993). Absence of rheumatoid arthritis in a rural Nigerian population. Journal of Rheumatology 20(4): 618-622.

51. Sumanth, S., Anusha and Swetha, S. (2012). Elucidation of mechanism of anti-arthritic action of Arthosansar - a polyherbal formulation. Indian Journal of Traditional Knowledge 11(4): 704-713.

52. Stasiuk, L.M., Abehsira, O. and Fournier, C. (1996). Collagen-induced arthritis in DBA/1 mice: cytokine gene activation following immunization with type II collagen. Cellular Immunology 173(1): 269-275.

53. Tatsuo, M.A.K.F., Carvalho, W.M., Silva, C.V., Miranda, A.E.G., Ferreira, S.H. and Francischi, J.N. (1994). Analgesic and anti-inflammatory effects of dipyrone in rat adjuvant arthritis model. Inflammation 18: 399-405.

54. Tona, L., Kambu, K., Ngimbi, N., Mesia, K., Penge, O., Lusakibanza, M., Cimanga, K., De Bruyne, T., Apers, S., Totté, J., Pieters, L. and Vlietinck, A.J. (2000). Antiamoebic and spasmolitic activities of extracts from some antidiarrhoeal traditional preparations used in Kinshasa, Congo. Phytomedicine 7: 31-38.

55. Vierboom, M.P., Jonker, M., Bontrop, R.E. and 'tHart, B. (2005). Modeling human arthritic diseases in non-human primates. Arthritis Research \& Therapy 7(4): 145-154.

56. Woolf, A.D., Pfleger, B., (2003). Burden of major musculoskeletal conditions. Bulletin of World Health Organization 81(9): 646-656.

57. World Health Organization (1996). W.H.O. guidelines for the assessment of herbal medicines. W.H.O. Expert Committee on specification for pharmaceutical preparations. Technical Report series No. 863. Geneva.

58. Yoshizaki, K., Nishimoto, N., Mihara, M. and Kishimoto, T. (1998). Therapy of rheumatoid arthritis by blocking IL-6 signal transduction with humanized anti-IL-6 receptor antibody. Springer Seminar Immunopathology 20: 247-259. 